\title{
Diseño y evaluación de un laboratorio virtual para visualizar momentos de un vector deslizante en 3D
}

\author{
José A. Gómez-Tejedor ${ }^{\mathrm{a}, \mathrm{b}}$, Juan A. Monsoriu ${ }^{\mathrm{a}, \mathrm{c}}$, Isabel Salinas ${ }^{\mathrm{a}, \mathrm{d}}$, Juan A. Sans ${ }^{\mathrm{a}, \mathrm{e}}$, \\ Vanesa P. Cuenca-Gotor ${ }^{\mathrm{a}, \mathrm{f}}$ y Marcos H. Giménez ${ }^{\mathrm{a}, \mathrm{g}}$ \\ ${ }^{a}$ ETS de Ingeniería del Diseño, Universitat Politècnica de València, bjogomez@fis.upv.es,

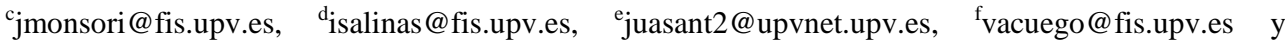 \\ gmhgimene@fis.upv.es.
}

\begin{abstract}
The use of multimedia tools for the development and implementation of teaching material is considered of paramount importance for first degree courses. Thus, we have observed, in fundamentals of Physics subjects, how the use of this kind of didactic material increases the motivation and the learning curve of the student. In this work, we present a virtual lab for the calculation of moments that allow multiple selections such as the vector representation, its action line and the moment in a $3 D$ environment. This applet allows the user modifying the point of view and the scale in an interactive way. Besides the visualization, this virtual lab allows the calculation of the moment of a sliding vector with respect to an axis and perform different operation between them. Additionally, it helps to understand the relation between the velocity at a point and the angular velocity of the rigid body in rotation to whom it belongs, the moment of a force with respect to an axis, and the way that this force affects the rotation. All of this supports the development of several transversal skills.
\end{abstract}

Keywords: Virtual laboratory, Physics, Easy Java, Sliding vector, moment.

\footnotetext{
Resumen

El uso de herramientas multimedia para el desarrollo e implementación de material didáctico se considera de una importancia capital para los primeros cursos de grado. Así, en las asignaturas de fundamentos de Física hemos observado cómo el uso de esta clase de material aumenta tanto la satisfacción como el aprendizaje del alumno. En este trabajo, presentamos un laboratorio virtual para el cálculo de momentos que permite múltiples opciones como la representación del vector, su línea de acción y su momento en un entorno 3D. Este applet permite al usuario modificar tanto el punto de vista como la escala de forma interactiva. Además de la visualización, este laboratorio virtual permite calcular el momento del vector deslizante respecto a un eje y realizar operaciones entre ellos. Asimismo, ayuda a comprender la relación entre la velocidad de un punto y la velocidad angular del sólido rígido en rotación al que pertenece, el momento de una fuerza
} 
respecto a un eje, y la forma en que dicha fuerza afecta a una rotación. Todo esto sustenta el desarrollo de diversas competencias transversales.

Palabras clave: Laboratorio Virtual, Física, Easy Java, Vector deslizante, momento.

\section{Introducción}

En la actualidad, las Tecnologías de la Información y la Comunicación (TICs) son ampliamente utilizadas en la enseñanza de las ciencias. Los métodos tradicionales de enseñanza, como es la lección magistral, están siendo reemplazados o compaginados con metodologías más activas enfocadas en los estudiantes. En este contexto, el docente actúa como un guía, brindando a los estudiantes las herramientas y mostrándoles las diferentes maneras en que pueden aplicarse al proceso de aprendizaje. El docente debe elegir la mejor manera de permitir que el alumno construya y comprenda la solución a problemas prácticos (Duffy, 1992). El docente no transfiere el conocimiento a los estudiantes, sino que los ayuda a construir, o al menos a interpretar, la realidad del cuerpo de conocimiento basado en sus experiencias.

En este contexto, los procesos de aprendizaje basados en simulaciones digitales han demostrado ofrecer varias ventajas (Jimoyainni, 2001; Gómez Tejedor, 2008), aunque su eficacia pedagógica está condicionada a una integración completa de la simulación en el plan de instrucción global (Vidaurre, 2002). La introducción de las TICs para la enseñanza tiene la ventaja de ofrecer la posibilidad de mostrar el tema de estudio desde diferentes puntos de vista. Concretamente, los laboratorios virtuales proporcionan a los estudiantes herramientas con las que controlar los parámetros relevantes de un fenómeno físico y visualizar las consecuencias (Depcik, 2005; Daineko, 2017; Lynch 2017).

En este trabajo presentamos un nuevo laboratorio virtual VISUALIZADOR DE MOMENTOS DE UN VECTOR DESLIZANTE. El laboratorio permite que el usuario introduzca las componentes de un vector deslizante y las coordenadas de un punto de su línea de acción. La aplicación calcula el momento del vector respecto a un punto seleccionado por el usuario. El vector, su línea de acción y su momento se muestran en una representación 3D en la que el usuario puede modificar de forma interactiva tanto el punto de vista como la escala. El laboratorio virtual también permite calcular el momento del vector deslizante respecto a un eje, además de representarlo gráficamente.

\section{Objetivos}

Con este laboratorio virtual, se pretende que los alumnos adquieran conocimientos y comprensión de diferentes conceptos relacionados con el cálculo del momento de un sistema de vectores respecto a un punto y respecto a un eje. En particular, los principales objetivos que el alumno puede alcanzar mediante la utilización de este laboratorio virtual son:

- Comprender y analizar cómo afectan a los momentos de un vector deslizante:

(cc) EY-NC-ND 2018, Universitat Politècnica de València

Congreso IN-RED (2018) 
José A. Gómez-Tejedor, Juan A. Monsoriu, Isabel Salinas, Juan A. Sans, Vanesa P. Cuenca-Gotor y Marcos H. Giménez

- El módulo, la orientación y la posición del vector.

- El desplazamiento del punto de aplicación del vector a lo largo de su línea de acción.

- El desplazamiento del punto respecto al que se calcula de forma paralela, radial o circular respecto a la línea de acción del vector deslizante.

- Analizar cómo afecta al momento respecto a un eje el desplazamiento del punto de dicho eje utilizado para el cálculo.

- Comprender la relación entre los momentos de la velocidad angular y las velocidades de los diferentes puntos de un sólido rígido en rotación.

- Comprender la relación entre el momento de una fuerza respecto a un eje y la forma en que dicha fuerza afecta a una rotación.

Estos objetivos se alcanzan tanto con la ayuda del profesor en el aula, como mediante un aprendizaje autónomo del alumno fuera de clase, trabajando así algunas de las competencias transversales establecidas por la Universitat Politècnica de València (UPV), como son: comprensión e integración; aplicación y pensamiento práctico; análisis y resolución de problemas; pensamiento crítico; y aprendizaje permanente.

\section{Desarrollo de la innovación}

\subsection{Definiciones}

Antes de entrar en la explicación del laboratorio virtual, es necesario realizar una serie de definiciones que se utilizan a lo largo de este trabajo y en el applet. Son definiciones que se explican en clase y que el alumno conoce, ya que este laboratorio virtual se utiliza precisamente para que el alumno trabaje y profundice en dichos conceptos.

Se define el momento de un vector deslizante $\vec{v}$ respecto a un punto $Q, \vec{M}(Q)$, como:

$$
\vec{M}(Q)=\overrightarrow{Q P} \times \vec{v}
$$

donde $P$ es un punto cualquiera de la línea de acción del vector $\vec{v}$ (Figura 1a).
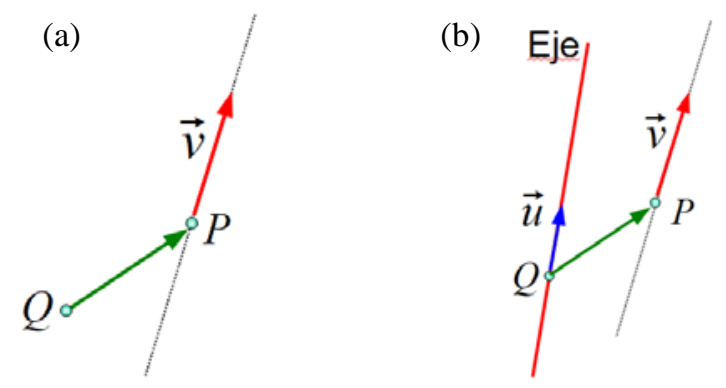

Fig. 1 (a) Momento de un vector respecto a un punto. (b) Momento de un vector respecto a un eje

Dado un eje definido mediante un punto $Q$ y un vector $\vec{w}$, se define el momento de un vector deslizante $\vec{v}$ respecto a dicho eje (momento áxico), $M(Q ; \vec{w})$, como:

$$
M(Q ; \vec{w})=\vec{M}(Q) \cdot \vec{u}=(\overrightarrow{Q P} \times \vec{v}) \cdot \vec{u}
$$


donde en esta expresión, $\vec{u}$ es un vector unitario en la dirección de $\vec{w}$, y define por tanto la dirección del eje (Figura 1b).

\subsection{Laboratorio virtual para la visualización de momentos de un vector deslizante}

Para alcanzar los objetivos descritos anteriormente, se ha desarrollado un laboratorio virtual VISUALIZADOR DE MOMENTOS DE UN VECTOR DESLIZANTE mediante la herramienta EASY JAVA SIMULATIONS, que se distribuye gratuitamente bajo la licencia de código abierto GNU GENERAL PUBLIC LICENSE. Dicha herramienta permite desarrollar aplicaciones basadas en el lenguaje de programación Java.

El applet permite que el usuario introduzca las componentes de un vector deslizante y las coordenadas de un punto de su línea de acción. La aplicación calcula el momento del vector respecto a un punto seleccionado por el usuario. El vector, su línea de acción y su momento se muestran en una representación 3D en la que el usuario puede modificar de forma interactiva tanto el punto de vista como la escala. Se puede acceder a la aplicación a través de la dirección web http://hdl.handle.net/10251/30531.

En la interfaz de la aplicación podemos destacar en primer lugar el menú Herramientas (Figura 2), que dispone de dos opciones: Restaurar vista 3D, que permite que el usuario pueda recuperar la perspectiva y escala iniciales; y Reiniciar, que devuelve el applet a su estado inicial.

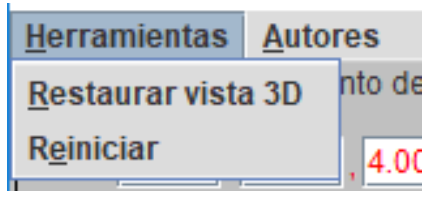

Fig. 2 Menú Herramientas.

En el panel Vector deslizante y punto de aplicación el estudiante puede introducir las tres componentes del vector deslizante $\vec{v}$ del cual se calcula el momento, y las coordenadas del punto de aplicación $P$ de dicho vector, utilizando para ello los cuadros de texto correspondientes (Figura 3). El programa calcula el módulo del vector, que se muestra a la derecha de sus componentes.

Conviene mencionar que la herramienta de desarrollo no permite poner flechas sobre los caracteres. Por este motivo, el laboratorio virtual muestra los vectores en negrita.

La aplicación calcula el momento de dicho vector respecto a un punto y/o un eje. En el panel Punto / eje para el cálculo de momentos, el usuario tiene que introducir las coordenadas del punto $Q$ respecto al que se quiere calcular el momento del vector, o un punto $Q$ y un vector $\vec{w}$ que definen la línea respecto a la cual se calcula el momento áxico (Figura 3). El programa calcula y muestra también el brazo $d$ del vector $\vec{v}$ respecto al punto $Q$. 
José A. Gómez-Tejedor, Juan A. Monsoriu, Isabel Salinas, Juan A. Sans, Vanesa P. Cuenca-Gotor y Marcos H. Giménez

A la hora de introducir los valores en los cuadros de texto anteriores hay que tener en cuenta que, al teclear cualquier entrada, dicho cuadro se muestra con fondo amarillo. Es obligatorio pulsar la tecla INTRO para validar la entrada, tras lo cual el fondo pasa a ser blanco. Si el valor introducido no es un número válido, el fondo cambia a color rojo, pudiendo entonces corregir la entrada.

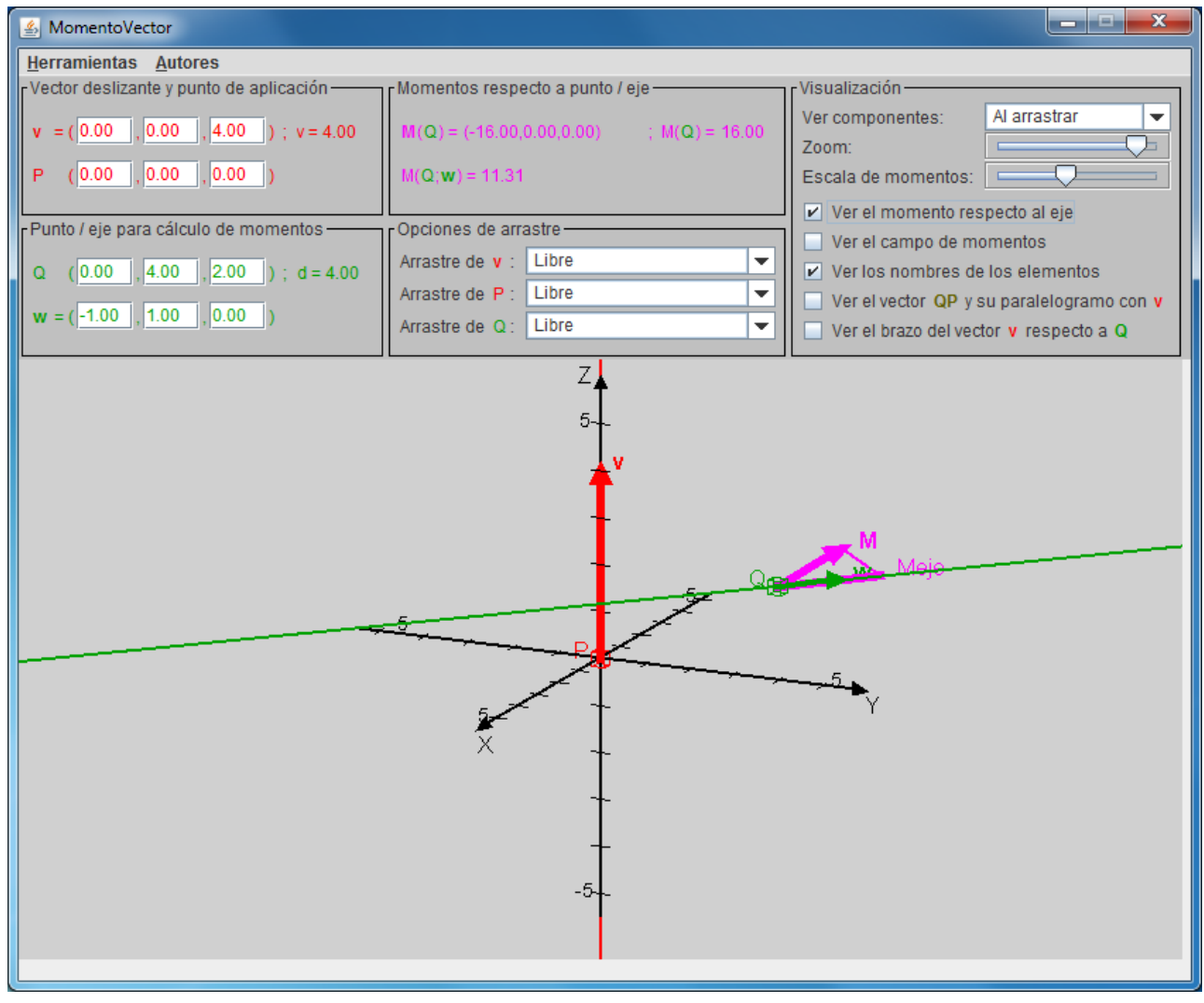

Fig. 3 Laboratorio virtual para la visualización de momentos de un vector deslizante

De forma alternativa, en lugar de introducir la información directamente con el teclado, el usuario puede modificar en el visor, arrastrando el ratón, tanto el vector como su punto de aplicación y el punto respecto al que se calcula el momento. De este modo, puede observar de forma interactiva cómo afectan esos elementos al momento resultante. Se puede elegir entre que esas modificaciones interactivas sean libres o estén sujetas a ciertos condicionantes de interés. Dado que el entorno mostrado en el visor es 3D, mientras que el ratón se mueve en 2D, no es posible modificar por arrastre las tres componentes de un vector, ni las tres coordenadas de un punto, de forma simultánea. Por ese motivo, solamente se modifican dos de ellas (cuáles en concreto depende del punto de vista utilizado). Sin embargo, es posible seleccionar una componente o coordenada cualquiera pulsando la tecla $\mathrm{X}, \mathrm{Y}$ o Z. Por ejemplo, si se arrastra el extremo de un vector mientras se mantiene pulsada la tecla $\mathrm{X}$, sólo se modifica esta componente. 
En el panel Momentos respecto a punto / eje se muestran los valores del vector momento respecto al punto y su módulo. Si está seleccionada la opción Ver el momento respecto al eje del panel Visualización se muestra también el momento respecto del eje.

En el panel Opciones de arrastre se puede controlar el comportamiento de la aplicación al seleccionar un punto o un vector, y arrastrarlo con el ratón. Al arrancar la aplicación aparece seleccionada la opción Libre en todas las listas desplegables, lo que permite mover libremente el vector $\vec{v}$, y/o los puntos $P$ y $Q$. Además de esta opción, según el caso, se dispone de las siguientes posibilidades:

- $\quad$ Arrastre de $\vec{v}$ :

- Módulo y sentido: permite cambiar el módulo y sentido del vector sin modificar su dirección (línea de aplicación del vector).

- Orientación: para cambiar la dirección del vector sin modificar su módulo.

- $\quad$ Arrastre de $P$ :

- Sobre línea de acción: desplaza el punto $P$, pero manteniéndolo siempre sobre la misma línea de acción del vector.

- $\quad$ Arrastre de $Q$ :

- Paralelo línea de acción: mueve el punto $Q$, manteniéndolo siempre en una línea paralela al vector $\boldsymbol{v}$.

- Radial línea de acción: el desplazamiento del punto $Q$ se realiza en una dirección perpendicular al vector $\vec{v}$.

- Circular línea de acción: desplaza el punto $Q$ siguiendo una circunferencia alrededor del vector $\vec{v}$. En este caso hay que tener en cuenta las restricciones del movimiento del ratón que es solamente en 2D, por lo que dependiendo de la perspectiva utilizada, no es posible describir la circunferencia completa alrededor del vector $\vec{v}$.

- Sobre el eje: esta opción solamente se puede utilizar cuando se visualiza el momento respecto al eje, seleccionando la opción correspondiente el panel de Visualización. En ese caso, la opción permite desplazar el punto $Q$ sobre el eje $(Q ; \vec{w})$.

Por último, mediante el panel Visualización se controlan varias opciones de la interfaz gráfica del applet. La opción Ver componentes hace que se muestren unos hilos (líneas verdes en el ejemplo de la Figura 4), que permiten visualizar más cómodamente la posición 3D Al arrastrar, Siempre o Nunca en función de la elección realizada. 


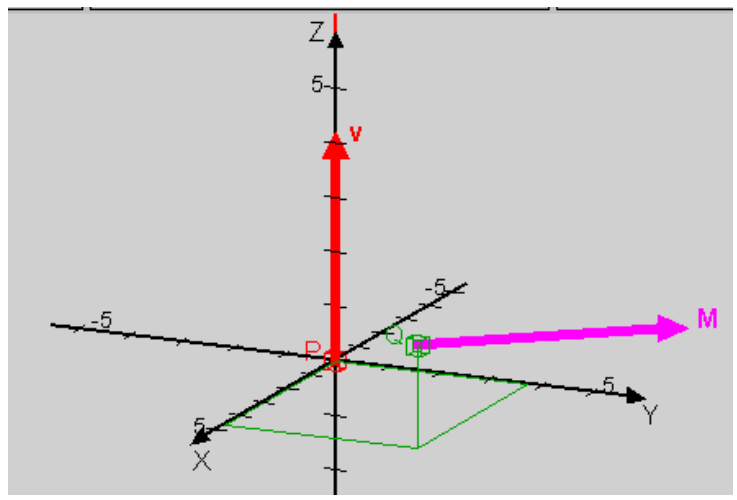

Fig. 4 Coordenadas del punto $Q$

La deslizadera Zoom permite ampliar o reducir el área visualizada en pantalla. La deslizadera Escala de momentos permite modificar la escala con que aparecen los momentos en el visor (téngase en cuenta que la magnitud del momento no es la misma que la de $\vec{v}$, por lo que no se corresponde con la graduación de los ejes del visor).

Por último, se proporciona una serie de opciones seleccionables mediante una casilla de verificación. Ver el momento respecto al eje (Figura 5a) permite ocultar y mostrar el momento respecto al eje. Ver el campo de momentos (Figura $5 \mathrm{~b}$ ) permite seleccionar entre visualizar o no el patrón de momentos alrededor del vector deslizante $\vec{v}$. Ver los nombres de los elementos permite elegir entre mostrar los nombres de los vectores y puntos junto a su representación gráfica, o bien ocultarlos para aclarar el visor. Ver el vector $Q P$ y su paralelogramo con $v$ (Figura 5 c) hace que se muestre en pantalla el paralelogramo definido por dichos vectores, lo que facilita ponerlo en relación con la definición de momento como un producto vectorial. Finalmente, Ver el brazo del vector $v$ respecto a $Q$ (Figura $5 \mathrm{~d}$ ) permite ver el brazo $d$ del vector deslizante respecto al punto, esto es, la distancia de $Q$ a la línea de acción de $\vec{v}$.

(a)

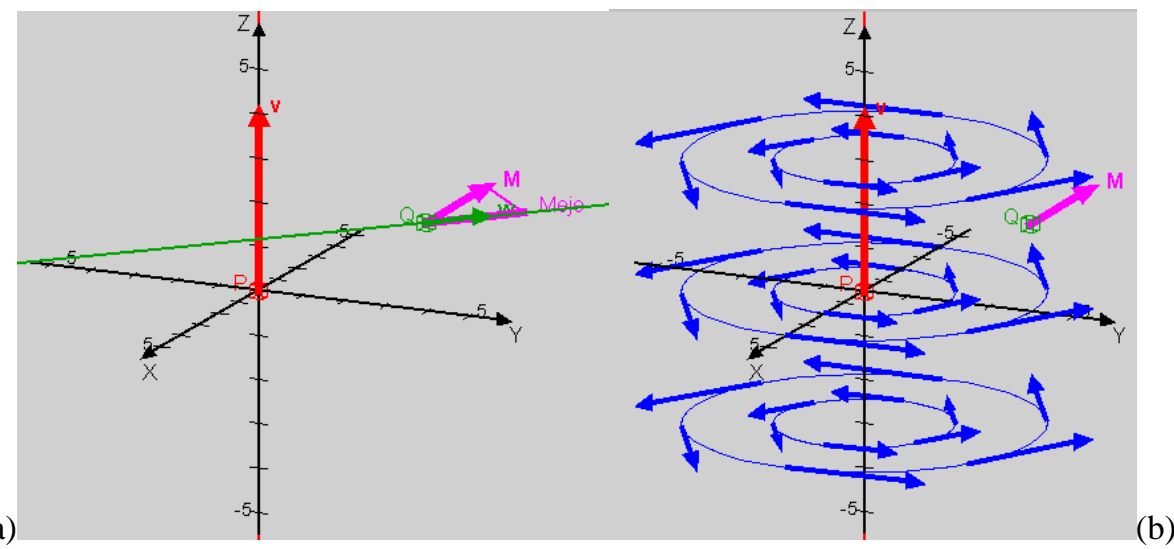

2018, Universitat Politècnica de València 


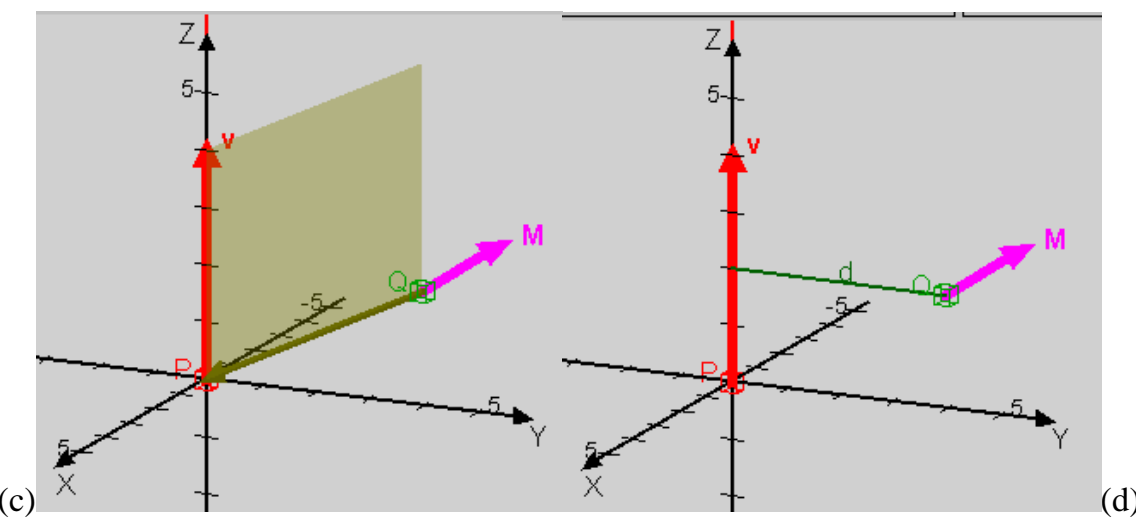

Fig. 5 Ejemplos del visor con diferentes opciones de visualización seleccionadas. (a) Ver el momento respecto al eje. (b) Ver el campo de momentos. (c) Ver el vector QP y su paralelogramo con v. (d) Ver el brazo del vector $v$

respecto a $Q$. En todos los casos está también seleccionada la opción de ver los nombres de los elementos

Por último, es interesante señalar que se utiliza un código de colores para identificar claramente cada uno de los elementos que se representan en el visor. El color es el mismo en el visor que en los diferentes paneles de la aplicación: rojo para el vector deslizante y su punto de aplicación, verde claro para el punto y eje para el cálculo de momentos, magenta para el momento, y azul para representar el campo de momentos.

\section{Resultados}

\subsection{Método de evaluación}

Con el objetivo de valorar la influencia del uso del laboratorio virtual "Visualizador de momentos de un vector deslizante" sobre el aprendizaje de los alumnos, durante el presente curso académico (2017/18) se han elegido dos grupos de control. Los dos grupos pertenecen al primer curso de dos títulos de grado de la Escuela Técnica Superior de Ingeniería del Diseño (ETSID) de la UPV y a dos asignaturas de física en las que figura en sus programas el tema de "Momentos de vectores deslizantes". Los grupos son:

- En el grupo de la asignatura "Complementos de Física" del grado en Ingeniería Mecánica se ha impartido el tema de Momentos utilizando el laboratorio virtual. Los alumnos de esta asignatura no tenían ningún conocimiento previo sobre el tema de Momentos. Por ello, una vez explicado el tema, se realizó un examen PoliformaT para valorar su aprendizaje.

- En la asignatura optativa "Física Básica” del grado en Ingeniería Eléctrica se ha impartido de forma clásica, sin utilizar el apoyo del laboratorio virtual. Los alumnos de esta asignatura optativa ya habían estado trabajando sobre este tema en la asignatura de "Física", por lo que se hizo un estudio comparativo entre los que habían cursado ambas asignaturas (grupo "Física Básica + Física”) y el resto de los estudiantes que solamente han cursado la asignatura de "Física” (grupo "Física”). 
José A. Gómez-Tejedor, Juan A. Monsoriu, Isabel Salinas, Juan A. Sans, Vanesa P. Cuenca-Gotor y Marcos H. Giménez

Para poder analizar el efecto del uso del laboratorio virtual sobre el rendimiento académico de los estudiantes se han realizado unas pruebas de respuesta numérica abierta. Asimismo, para conocer cuál ha sido el grado de satisfacción respecto a la utilización del laboratorio virtual se ha realizado una encuesta anónima a los alumnos de la asignatura "Complementos de Física". La encuesta plantea ocho cuestiones y las respuestas son valoradas con cinco niveles (TDA: totalmente en desacuerdo; DA: en desacuerdo; IND: indiferente; A: de acuerdo; TA: totalmente de acuerdo).

\subsection{Test de rendimiento académico}

En primer lugar, al comparar los resultados obtenidos en las pruebas realizadas, encontramos que el valor medio de las puntuaciones es muy dispar. La nota media en "Complementos de Física" (utilizando el laboratorio virtual) ha sido de 9,07 con una desviación estándar de 1,17 (figura 6a), mientras que en el caso de "Física Básica" (sin utilizar el laboratorio virtual) la nota media ha sido de 6,74 con una desviación estándar de 2,08 (figura 6b).

Analizando los resultados obtenidos por los alumnos que utilizaron el laboratorio virtual, podemos observar que el poder interactuar con los parámetros que afectan al momento de un vector deslizante mejora el nivel de comprensión del tema abordado. Así mismo, a partir de la experiencia de los profesores en el aula, podemos afirmar que el nivel de motivación es muy alto.

Por otra parte, los alumnos que solamente cursaron la asignatura "Física" obtuvieron una nota media de 6,38 con una desviación estándar de 2,21. Si comparamos estos resultados con los de los alumnos que han recibido una clase de refuerzo (grupo "Física Básica + Física"), podemos observar que estos últimos han mejorado la nota media, como se muestra en la figura $6 \mathrm{~b}$.

\section{Complementos de Física}
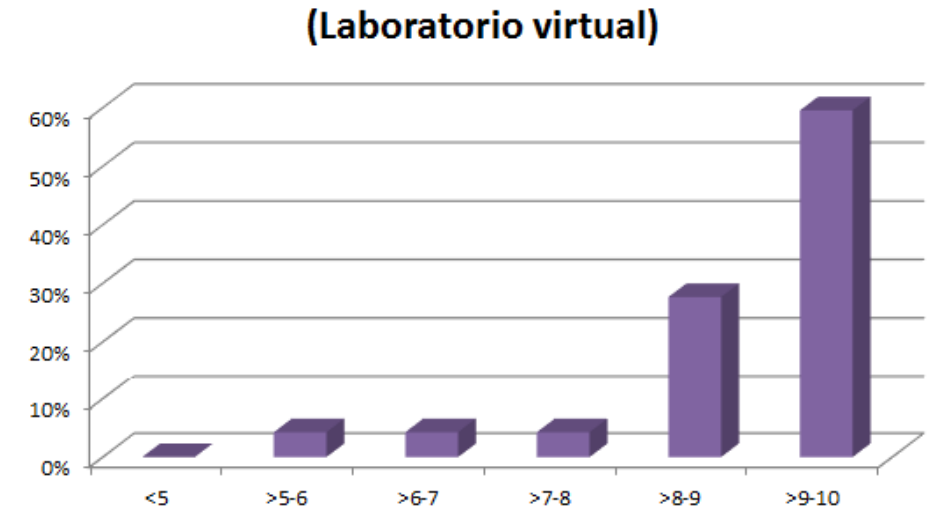

Fig. 6a Representación gráfica del porcentaje de alumnos que han obtenido una determinada calificación en la asignatura "Complementos de Física" donde se ha utilizado el laboratorio virtual. 


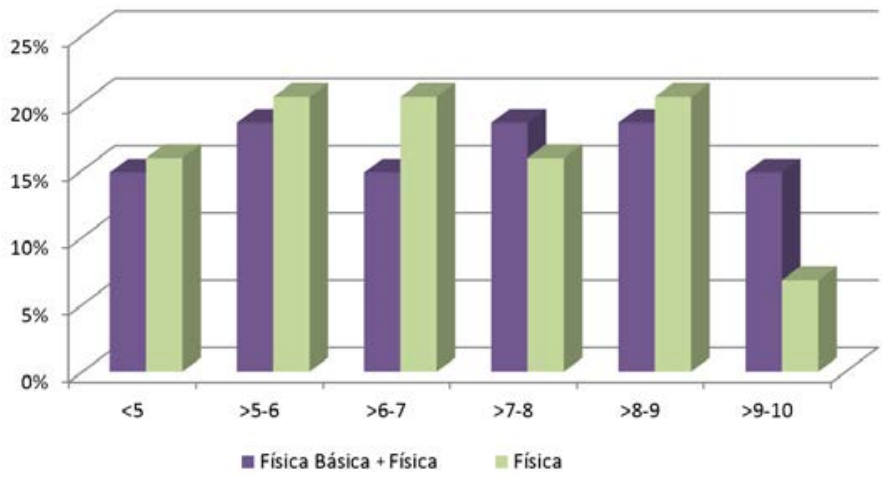

Fig. 6 b Representación gráfica del porcentaje de alumnos que han obtenido una determinada calificación en los exámenes de los grupos "Física Básica+Física" y "Física".

\subsection{Encuesta de opinión}

La encuesta se ha realizado en el grupo de estudiantes que ha utilizado el labora-torio virtual. En la Tabla 1 se muestran las preguntas y las respuestas obtenidas sobre una muestra de 34 alumnos.

Las respuestas de las cuestiones que están relacionadas con la manejabilidad del laboratorio virtual nos dicen que más del $76 \%$ de los estudiantes exploró las opciones que esta herramienta ofrece (Q6), y el 88,2\% encontró que el manejo del laboratorio virtual era intuitivo y sencillo (Q2).

La percepción del estudiante sobre la mejoría experimentada en la comprensión del tema de Momentos, después de haber utilizado el laboratorio virtual, es también muy positiva ya que el 82,4\% manifiesta haber mejorado (Q4) y más del 94\% que le ha sido útil (Q1).

Tabla 1. Preguntas y resultados de la encuesta de opinión

\begin{tabular}{|c|l|c|c|c|c|c|}
\hline \multicolumn{2}{|l|}{ PREGUNTA } & TDA & DA & IND & A & TA \\
\hline Q1 & $\begin{array}{l}\text { Me ha resultado útil el laboratorio virtual } \\
\text { "Visualizador de momentos de un vector deslizante". }\end{array}$ & 0 & 0 & 2 & 10 & 22 \\
\hline Q2 & $\begin{array}{l}\text { Me ha resultado fácil e intuitivo el manejo del } \\
\text { laboratorio virtual "Visualizador de momentos de un } \\
\text { vector deslizante". }\end{array}$ & 0 & 0 & 4 & 20 & 10 \\
\hline Q3 & $\begin{array}{l}\text { En general, mi nivel de comprensión de los conceptos } \\
\text { antes de utilizar el laboratorio de "Visualizador de } \\
\text { momentos de un vector deslizante" era bueno. }\end{array}$ & 0 & 4 & 8 & 18 & 4 \\
\hline Q4 & $\begin{array}{l}\text { Mi nivel de comprensión de los conceptos después de } \\
\text { utilizar el laboratorio virtual "Visualizador de } \\
\text { momentos de un vector deslizante" ha mejorado }\end{array}$ & 0 & 0 & 6 & 14 & 14 \\
\hline
\end{tabular}


José A. Gómez-Tejedor, Juan A. Monsoriu, Isabel Salinas, Juan A. Sans, Vanesa P. Cuenca-Gotor y Marcos H. Giménez

\begin{tabular}{|l|l|c|c|c|c|c|}
\hline & respecto a mi nivel previo. & & & & & \\
\hline Q5 & $\begin{array}{l}\text { Me resulta más motivador repasar el tema "Momento } \\
\text { de un vector deslizante" utilizando el laboratorio } \\
\text { virtual de "Visualizador de momentos de un vector } \\
\text { deslizante", que revisar comentarios aportados por el } \\
\text { profesor (apuntes, notas, diapositivas...) }\end{array}$ & 0 & 2 & 6 & 20 & 6 \\
\hline Q6 & $\begin{array}{l}\text { Cuando he utilizado el laboratorio virtual de } \\
\text { "Visualizador de momentos de un vector deslizante" } \\
\text { he explorado muchas de las opciones que ofrece. }\end{array}$ & 0 & 2 & 6 & 14 & 12 \\
\hline Q7 & $\begin{array}{l}\text { Mi valoración general del laboratorio virtual de } \\
\text { "Visualizador de momentos de un vector deslizante" } \\
\text { es buena. }\end{array}$ & 0 & 0 & 0 & 20 & 14 \\
\hline Q8 & $\begin{array}{l}\text { El laboratorio de "Visualizador de momentos de un } \\
\text { vector deslizante" ha contribuido a mejorar mi } \\
\text { habilidad para analizar y resolver problemas. }\end{array}$ & 0 & 2 & 8 & 14 & 10 \\
\hline
\end{tabular}

Dada la importancia de la motivación en el proceso de aprendizaje se analiza la pregunta Q5, que está vinculada con este factor. Esta cuestión trata de indagar sobre el efecto motivador del laboratorio virtual comparado con el material aportado por el profesor de una forma más tradicional, como apuntes, notas, diapositivas, etc. En la Figura 7 se muestra mediante un gráfico qué porcentaje de los estudiantes ha respondido en cada uno de los cinco niveles utilizados para valorar la pregunta. Observamos que un porcentaje muy elevado, el 77\%, ha encontrado más motivadora la utilización del laboratorio frente a un $23 \%$ que, o bien no lo ha considerado así en absoluto, 6\%, o no ha apreciado diferencia, $17 \%$.

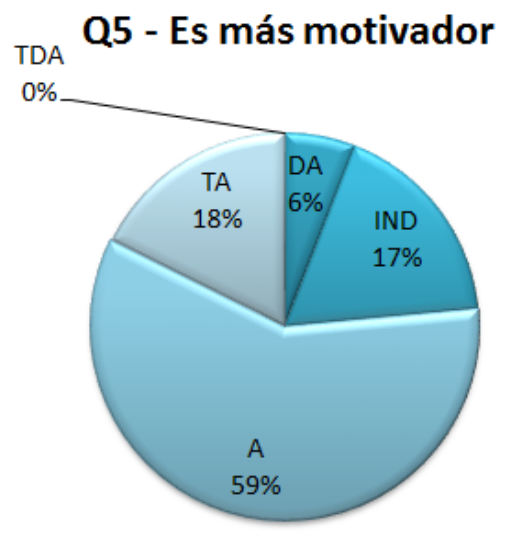

Fig. 7 Representación gráfica del porcentaje de alumnos que ha respondido en cada uno de los cinco niveles de la pregunta Q5. 
La pregunta Q7 da respuesta a la valoración general del laboratorio. Como se puede observar en el gráfico de la Figura 8, la valoración de los alumnos es muy positiva ya que el $100 \%$ está de acuerdo o totalmente de acuerdo con la afirmación de que es bueno.

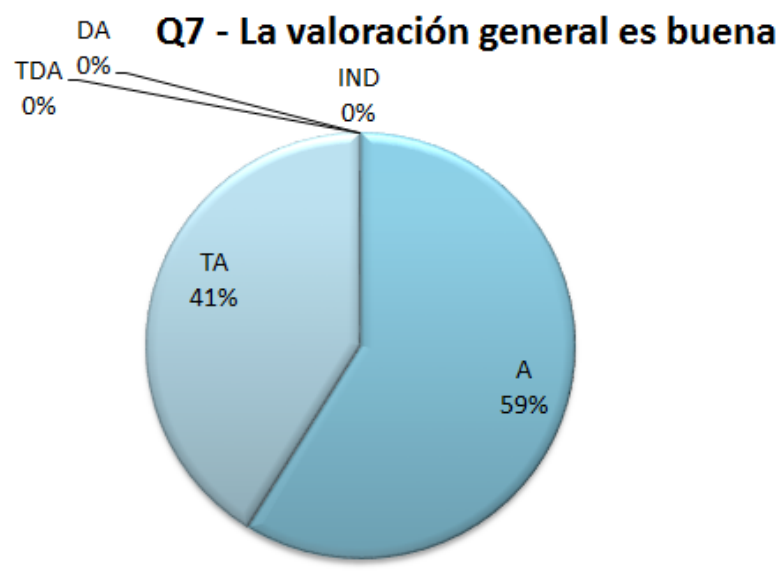

Fig. 8 Representación gráfica del porcentaje de alumnos que ha respondido en cada uno de los cinco niveles de la pregunta Q7.

\subsection{Competencias transversales}

Este laboratorio proporciona a los estudiantes los medios para desarrollar su propia comprensión sobre el tema de cálculo, visualización y aplicación práctica de los momentos de vectores deslizantes. El profesor actúa como guía, proporcionando a los estudiantes las herramientas y mostrándoles las diferentes formas en que pueden aplicarse al proceso de aprendizaje. De este modo, el laboratorio virtual que presentamos sirve de herramienta para la obtención de varias competencias transversales clave de las COMPETENCIAS TRANSVERSALES UPV, que ayudarán al alumno a tener una formación más completa para su futuro profesional. El estudiante debe:

- Demostrar la comprensión e integración de los conocimientos, tanto en la propia especialización como en otros contextos más amplios (CT1-Comprensión e Integración).

- Aplicar los conocimientos a la práctica, atendiendo a la información disponible y estableciendo el proceso a seguir para alcanzar los objetivos con eficacia y eficiencia (CT2Aplicación y Pensamiento Práctico).

- Realizar tareas que requieren procesos de razonamiento más o menos complejos y, en muchos casos, no simplemente una acción asociativa y de rutina. También debe identificar y definir los elementos significativos de un problema para su análisis y resolución efectivos (CT3-Análisis y Resolución de Problemas).

- Desarrollar un pensamiento crítico, interesado en las bases sobre las que se basan las ideas, acciones y juicios, tanto propios como ajenos. Esto implica cuestionar las suposicio-nes que subyacen a nuestras formas habituales de pensar y actuar (СT9Pensamiento Crítico). 
José A. Gómez-Tejedor, Juan A. Monsoriu, Isabel Salinas, Juan A. Sans, Vanesa P. Cuenca-Gotor y Marcos H. Giménez

- Utilizar el aprendizaje de manera estratégica, autónoma y flexible, para manipular el conocimiento, actualizarlo y seleccionar lo que es apropiado para un contexto específico en función del objetivo perseguido (CT11-Aprendizaje Permanente).

El estudiante debe prepararse para enfrentar situaciones complejas acomodando los recursos disponibles. El uso de estas herramientas (laboratorios virtuales) promueve la autonomía y la iniciativa. El estudiante aprende y aplica conocimientos y habilidades de manera autónoma, de esta forma mejora su capacidad de analizar y resolver problemas (Figura 9).

\section{Q8 - Mejora la capacidad de analizar y resolver problemas}

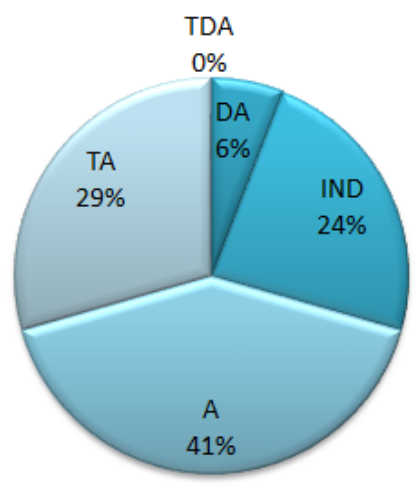

Fig. 9 Representación gráfica del porcentaje de alumnos que ha respondido en cada uno de los cinco niveles de la pregunta Q8

\section{Conclusiones}

El desarrollo de un laboratorio virtual sobre el tema de cálculo, visualización y aplicación práctica de los momentos de vectores deslizantes ha demostrado ser una herramienta muy útil para incrementar el aprendizaje de los alumnos y su motivación, en los grupos de estudio de este trabajo. Pensamos que esta metodología de visualización en una representación tridimensional de determinadas magnitudes puede ser aplicada a otros temas tanto de física como de otras asignaturas. Este tipo de metodología puede facilitar también el trabajo de algunas competencias transversales de forma más sencilla que mediante una metodología clásica.

\section{Agradecimientos}

JAS agradece al programa Ramón y Cajal la financiación y al Instituto de Diseño para la Fabricación y Producción Automatizada (IDF-UPV) por su apoyo. 


\section{Referencias}

DAINEKO, Y., DMITRIYEV, V. y IPALAKOVA, M. (2017). "Using virtual laboratories in teaching natural sciences: An example of physics courses in university" en Computer Applications in Engineering Education 25, 39-47.

DEPCIK, C. y ASSANIS, D.N. (2005). "Graphical user interfaces in an engineer in educational environment” en Computer Applications in Engineering Education, 13, 48-59.

DUFFY, T. y JONASSEN, D. (1992) Constructivism and the technology of instruction. Hillsdale, New Jersey: Lawrence Erlbaum Associates.

Easy Java Simulations. <http://fem.um.es/Ejs/> [Consulta: 13 de marzo de 2018].

GNU General Public License. <http://www.gnu.org/copyleft/gpl.html> [Consulta: 13 de marzo de 2018].

GÓMEZ TEJEDOR, J.A., MOLTÓ MARTÍNEZ, G. y BARROS VIDAURRE, C. (2008) “An Online Virtual Laboratory of Electricity” en International Journal of Distance Education Technologies, 6 (2), 21-34.

JIMOYAINNI, A. y KOMIS, V. (2001). “Computer simulations in physics teaching and learning” en Computers \& Education, 36, 183-204.

LYNCH T. y GHERGULESCU I., (2017) "NEWTON Virtual Labs: Introduction and Teacher Perspective” en 17th International Conference on Advanced Learning Technologies (ICALT), IEEE, 343-345.

SALINAS MARÍN, I., GIMÉNEZ VALENTÍN, M.H. y MONSORIU SERRA, J.A. Visualizador de momentos de un vector deslizante (2013). <http://hdl.handle.net/10251/30531> [Consulta: 13 de marzo de 2018].

UNIVERSITAT POLITÈCNICA DE VALÈNCIA. Competencias Transversales UPV (2012). $<$ http://www.upv.es/contenidos/COMPTRAN/> [Consulta: 23 de marzo de 2018]

VIDAURRE, A., RIERA, J., GIMÉNEZ, M.H. y MONSORIU, J.A. (2002). “Contribution of digital simulation in visualizing physics processes” en Comput. Appl. Eng. Educ. 10(1), 45-49. 